\title{
TOPOLOGICAL QUANTUM CHARACTERISTICS OBSERVED IN THE INVESTIGATION OF THE CONDUCTIVITY IN NORMAL METALS
}

\author{
S. P. NOVIKOV AND A. YA. MAL'TSEV
}

\begin{abstract}
It is shown that the investigation of the conductivity in a single crystal of a normal metal with a complicated Fermi surface in strong magnetic fields B can reveal integral topological characteristics which are determined by the topology of open-ended quasiclassical electron trajectories. Specifically, in the case of open-ended trajectories of the general position there always exists a direction $\eta$ orthogonal to $\mathbf{B}$ in which the conductivity approaches zero for large $B$, and this direction lies in some integral (i.e., generated by two reciprocal-lattice vectors) plane that remains stationary for small variations of the direction of $\mathbf{B}$.
\end{abstract}

In the present letter we describe topological effects arising in the study of the conductivity tensor of normal metals in strong magnetic fields. We assume that conductivity is described well in the quasiclassical approximation for the one-electron problem with some dispersion law $\epsilon(\mathbf{p})$, which is periodic in the quasimomentum space with periods equal to reciprocal-lattice vectors. The only vestiges of quantum mechanics in the theory are a transition from Euclidean momentum space $\mathcal{E}=R^{3}$ to the first Brillouin zone $\mathcal{B}$, which is a three-dimensional torus $T^{3}$ related with the reciprocal lattice, and the form of the function $\epsilon(\mathbf{p})$. In this approximation the electrons follow quasiclassical trajectories which, as functions of the time $t$, are solutions of the system

$$
\frac{d \mathbf{x}}{d t}=\nabla \epsilon(\mathbf{p}), \quad \frac{d \mathbf{p}}{d t}=\frac{e}{c}[\nabla \epsilon(\mathbf{p}) \times \mathbf{B}] .
$$

The system (1) is a Hamiltonian system with the Hamiltonian $H(\mathbf{x}, \mathbf{p})=\epsilon(\mathbf{p})$ and Poisson brackets $\left\{x_{i}, x_{j}\right\}=0,\left\{x_{i}, p_{j}\right\}=\delta_{i j},\left\{p_{i}, p_{j}\right\}=e B_{i j} / c(i=1,2,3)$, where $B_{i j}=-B_{j i}, B_{23}=B_{1}, B_{31}=B_{2}$, and so on. The trajectories of the system (1) in momentum space are given by the intersection of the surfaces of constant energy $\epsilon(\mathbf{p})=$ const with planes perpendicular to the magnetic field:

$$
\mathbf{p} \cdot \mathbf{B}=p_{j} B_{j}=\text { const. }
$$

The theory described above works well up to the comparatively high values of the magnetic field $B$ which we shall study. For comparison we point out that the upper limit on the magnetic field is determined by the applicability of the quasiclassical approximation $\omega_{B} \ll \epsilon_{F}$, while the effects due to the topology of the trajectories come into play for $\omega_{B}>\tau^{-1}$, where $\tau$ is the free time of flight of the electrons (see $[1]-[3])$.

Date: Submitted 17 April 1996.

PACS numbers: $72.15 . \mathrm{Cz} ; 72.15 . \mathrm{Eb}$

Pis'ma Zh. Éksp. Teor. Fiz. 63, No. 10, 809-813 (25 May 1996). Translated by M. E. Alferieff. 
Although the system can be integrated analytically in the expanded Brillouin zone $\mathcal{E}$, nontrivial topological effects are possible in the case of complicated Fermi surfaces. In this case open trajectories, defined as open-ended in $\mathcal{E}$, can exist. If such a trajectory is closed in $\mathcal{B}$, we shall call it open periodic.

It is well known that if all trajectories of the system (1) are closed, then the electrical conductivity in a plane $\Pi$ perpendicular to the magnetic field $\mathbf{B}$ approaches zero as $B \rightarrow \infty$. As a result of the existence of open-ended trajectories, terms which do not vanish as $B \rightarrow \infty$ can appear in the conductivity tensor $\sigma_{\alpha \beta}(\alpha, \beta=1,2)$ in the plane $\Pi$. If all such trajectories lie in bands of finite width, having (in all planes orthogonal to $\mathbf{B}$ and at all energy levels in which open trajectories exist) the same general direction $\vec{\eta}(\mathbf{B})$, then the tensor $\sigma_{\alpha \beta}$ has a very simple structure in the limit $B \rightarrow \infty: j_{\vec{\eta}}=0, j_{\zeta}=\sigma_{\zeta} E_{\zeta}$. Here $\mathbf{j}$ is the current in the plane $\Pi, \mathbf{E}$ is the electric field, $\zeta$ is a direction perpendicular to $\vec{\eta}$ in the plane $\Pi$, i.e., crap is a degenerate tensor with kernel $\vec{\eta}(\mathbf{B})$. These results all follow naturally from the contributions of the individual trajectories, described in [1]-[3]. A more detailed discussion can be found in [4] (as indicated to the authors by V. G. Peschanskiur, this book contains experimental observations related with the results of the present work).

The main result of the present work is that if open trajectories exist for a field $\mathbf{B}$ with an irrational direction (i.e., such that the plane $\Pi$ does not contain reciprocallattice vectors), then the situation described above occurs necessarily and therefore the above-described form of the conductivity tensor in the limit $B \rightarrow \infty$, if it does not vanish, is the only possible one. Moreover, in this case it can be asserted that open-ended trajectories exist and possess the same properties as for magnetic fields with directions lying in some open neighborhood of the irrational initial direction (on the unit sphere), the above-described direction $\vec{\eta}(\mathbf{B})$ being given each time by the intersection of $\Pi(\mathbf{B})$ with a integral (i.e., generated by two reciprocal-lattice vectors) plane $\Gamma\left(\mathbf{B}_{0}\right)$, which is the same for a given neighborhood.

The assertion formulated above describes the most general situation, i.e., the case of magnetic fields of the general position. The experimentally observed zones of stability and the corresponding integral planes $\Gamma\left(\mathbf{B}_{0}\right)$ are nontrivial topological characteristics of a metal with a complicated Fermi surface. If the magnetic field is such that the plane $\Pi(\mathbf{B})$ contains reciprocal-lattice vectors, the assertion that each open trajectory lies in a band of finite width and passes through it remains valid. However, besides the above described trajectories "of the general position," open trajectories with asymptotic directions along the reciprocal-lattice vectors $\vec{\eta}_{\alpha}$ contained in $\Pi(\mathbf{B})$ can also arise. Each family of such trajectories makes in the limit $B \rightarrow \infty$ a nonvanishing contribution to $\sigma_{\alpha \beta}$ that is identical to the above-described contribution with the only difference that now $\vec{\eta}_{\alpha}, \boldsymbol{\zeta}_{\alpha}$, and $\sigma_{\zeta \alpha}$ appear instead of $\vec{\eta}, \boldsymbol{\zeta}$, and $\sigma_{\zeta}$, respectively. The number of integral directions for which such a situation is observed in the metal does not exceed the genus $g$ of the Fermi surface, ${ }^{1}$ so that the direction of the magnetic field must be specially chosen so as to be able to observe such contributions to the conductivity tensor. These contributions are easy to distinguish experimentally, since the corresponding open trajectories remain only for variations of $\mathbf{B}$ which are perpendicular to the corresponding $\vec{\eta}_{\alpha}$ and they vanish for all other variations, which gives rise to a jump in the tensor $\sigma_{\alpha, \beta}$. It also

\footnotetext{
${ }^{1}$ Any two-dimensional closed orientable surface is topologically equivalent to a two-dimensional sphere with "handles." The genus $g$ of the surface equals the number of handles.
} 
follows from the topological analysis that if together with such trajectories for a given direction of the magnetic field the above-described trajectories of the general position also exist, then the vectors $\vec{\eta}_{\alpha}$ observed in this case should lie in the $\Gamma(\mathbf{B})$ plane.

Therefore the observation of electrical conduction in metals with a complicated Fermi surface in strong magnetic fields makes it possible to determine the following:

1) the integral directions $\vec{\eta}_{\alpha}$ and

2) the above-described zones of stability with the corresponding integral planes $\Gamma\left(\mathbf{B}_{0}\right)$.

The physical consequences formulated above were obtained from the mathematical results presented in $[5]-[8]^{2}$ (see also Refs. 9 and 10). We shall present these results here.

Using the starting point $\mathbf{p}(0)$ on an open trajectory, we shall call the limits

$$
\vec{\eta}_{ \pm}=\lim _{t \rightarrow \pm \infty}[\mathbf{p}(t)-\mathbf{p}(0)] /|\mathbf{p}(t)-\mathbf{p}(0)|,
$$

if they exist, outgoing and incoming asymptotic directions, respectively.

Further, if $\vec{\eta}_{+}=-\vec{\eta}_{-}=\vec{\eta}$, we shall say that the open trajectory has an average direction. If, in addition, the trajectory lies in a strip of finite width in the plane (2) (necessarily parallel to $\vec{\eta}$ ), then we shall say that it possesses a strong average direction.

In describing the implications of the topological theorems, formulated mainly in Refs. 6 and 8, we shall assume for simplicity that $\nabla \epsilon(\mathbf{p})$ does not vanish on the energy levels considered (i.e., we exclude the energies at which an isoenergy level is restructured) and that each special trajectory contains a single special point (this holds for fields of the general position).

There are two possibilities:

1) In very special cases it can happen that open trajectories which do not have an average direction exist on one energy level (see Ref. 10). In this case all trajectories are closed on all other energy levels. This situation corresponds to zero phase volume and does not contribute to the conductivity.

2) In the case of the general position [i.e., if Eq. (1) does not hold] for fields with an irrational direction open trajectories, if they exist, exist on energy levels in some connected energy interval $\epsilon_{1}<\epsilon<\epsilon_{2}$, and they all have a strong average direction, given by the intersection of the plane $\Pi(\mathbf{B})$, perpendicular to the magnetic field, by some locally stable integral plane $\Gamma\left(\mathbf{B}_{0}\right)$. There are no open trajectories on levels lying outside this interval. If the Fermi level falls within the indicated interval, then the contribution of such trajectories with remarkable topological properties to the conductivity is observed experimentally.

In the more general case, i.e., not requiring that the directions of $\mathbf{B}$ be irrational, the assertion is that any open trajectory lies in the Brillouin zone $\mathcal{B} \equiv T^{3}$ on a twodimensional torus of the $M$ or $N$ type (membrane and needle, respectively). We shall describe these concepts.

For a fixed direction of the magnetic field, we remove from each energy level in $\mathcal{B}$ those parts which consist of nonsingular trajectories that are closed in $\mathcal{E}$. The boundary of the manifold obtained will be singular orbits which are closed in $\mathcal{E}$ and which can be glued in $\mathcal{B}$ by disks lying in the plane $\Pi(\mathbf{B})$. A nontrivial

\footnotetext{
${ }^{2}$ The topological problem is formulated in [5] and some questions present in [5] are made more precise in [7].
} 


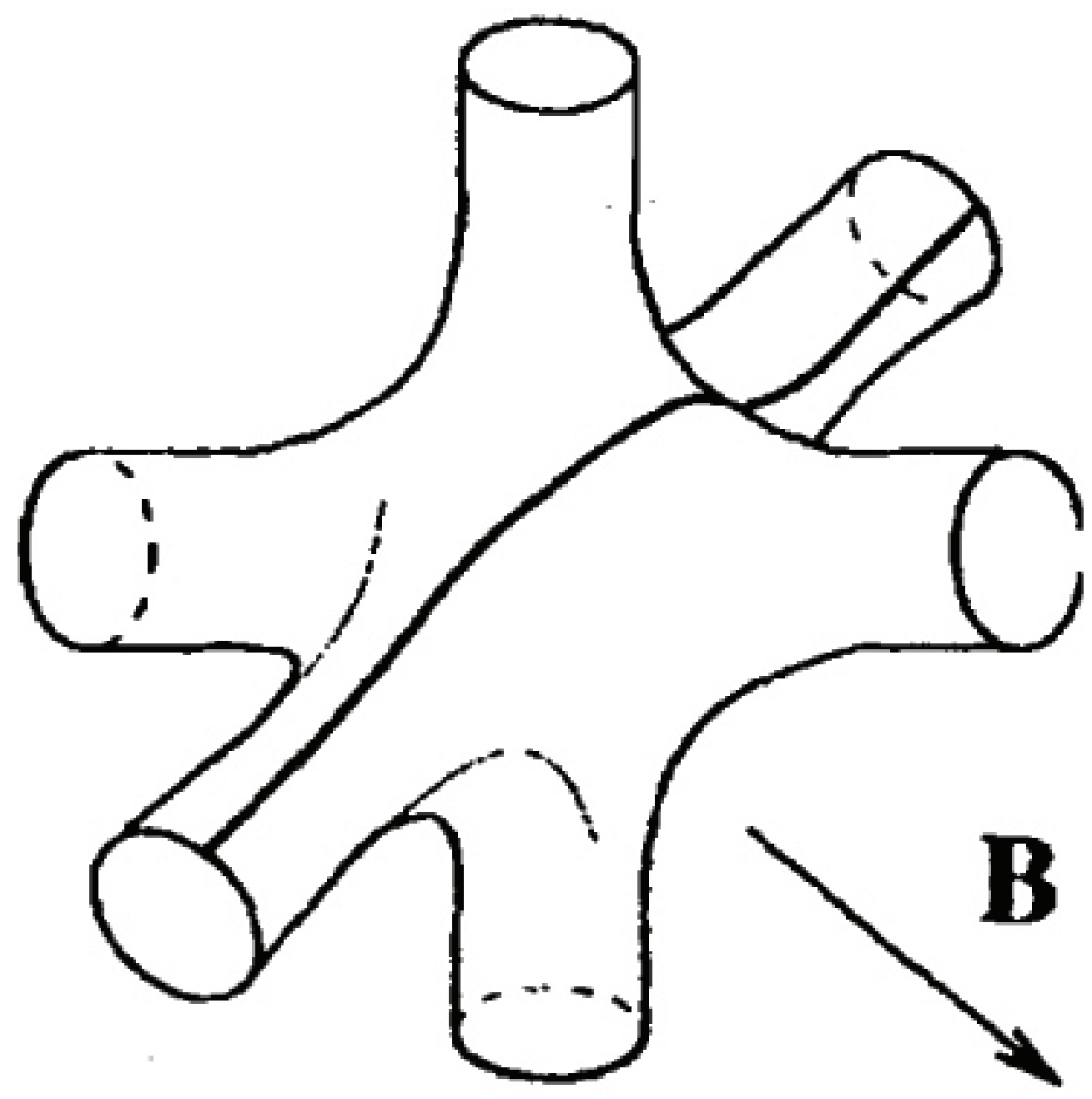

FigURE 1. Open trajectory corresponding to the integral direction $(1,0,0)$ in the plane $\Pi(\mathbf{B})$ perpendicular to the magnetic field

topological theorem (see [8]) asserts that the manifold obtained is a disjoint union of nonintersecting two-dimensional tori in $\mathcal{B}$; two-dimensional tori lying on different energy levels also do not intersect one another.

Two types of two-dimensional tori can appear. The simplest type is a "needle" or a $N$ type torus, bounding the region in $\mathcal{B}$ and topologically homologous to zero in $\mathcal{B}$. The embedding of such a torus in $\mathcal{B}$ is reminiscent of a cylinder or tube and can be continuously deformed into a closed curve in $\mathcal{B}$. In an expanded zone $\mathcal{E}$ the covering of such a torus is an infinite periodically-deformed ("fluted") cylinder, determining a reciprocal-lattice $\vec{\eta}_{\alpha}$. The longitudinal sections of this cylinder by the plane $\Pi(\mathbf{B})$ give open trajectories in $\mathcal{E}$. It is obvious that tori of this type can arise only if $\Pi(\mathbf{B})$ contains reciprocal-lattice vectors and they vanish for variations 


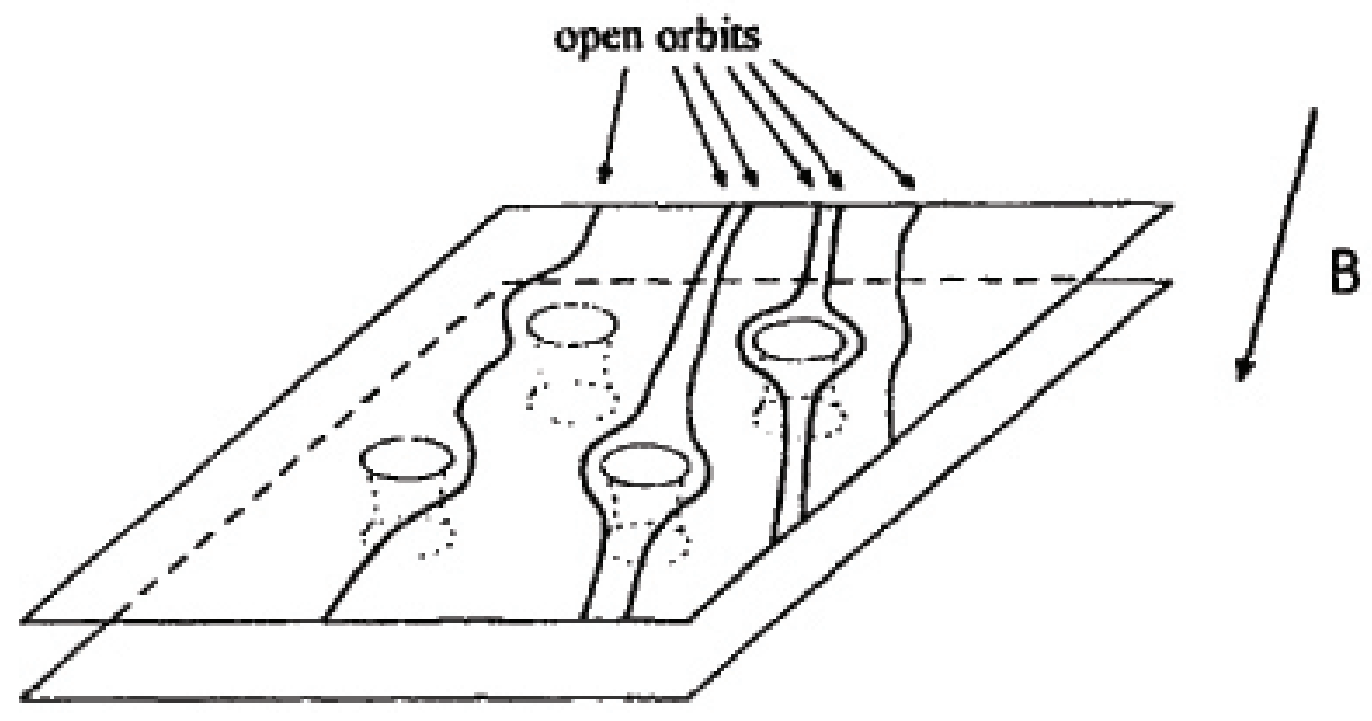

Figure 2. Pair of integral planes in the complicated Fermi surface. The pair is obtained after the closed trajectories are removed. The open trajectories are given by the intersection of these planes by a plane perpendicular to the magnetic field.

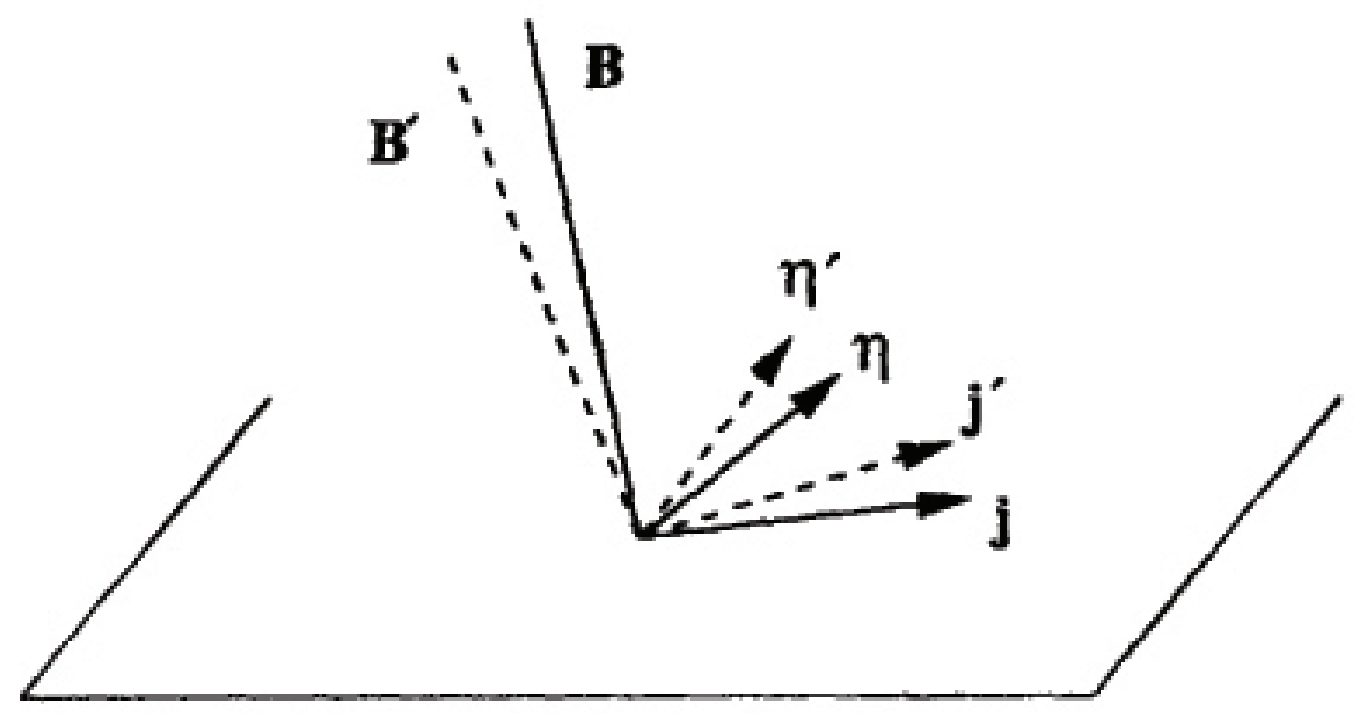

Figure 3 . The conductivity vanishes in the direction $\vec{\eta}$ perpendicular to $\mathbf{B}$ in the limit $B \rightarrow \infty$. The direction $\vec{\eta}$ is perpendicular to the current $\mathbf{j}$ observed in the limit $B \rightarrow \infty$. 
of $\mathbf{B}$ that destroy this property. A trivial case in which the open trajectories are directed along the $p_{x}$ axis is shown in Fig. 1.

The other possibility is a "membrane" or $M$ type torus, which is not homologous to zero in $\mathcal{B}$ (i.e., it does not bound any region in $\mathcal{B}$ ). In the expanded Brillouin zone its covering is a periodically deformed integral plane ("fluted plane"), whose intersection with the plane $\Pi(\mathbf{B})$ gives the above-described open trajectories of the "general position." All such planes corresponding to all type $M$ tori are parallel in $\mathcal{E}$, since they do not intersect one another, and their common homology class is the previously described integral plane $\Gamma\left(\mathbf{B}_{0}\right)$. The closure of an open trajectory lying on a type $M$ torus in $\mathcal{B}$ covers this torus, except for the initial openings, with $\alpha(\mathbf{B})$ revolutions. Thus such a trajectory can be regarded as quasiperiodic and therefore for isoenergetic surfaces of the general position any open trajectory is either periodic or quasiperiodic. The number of type $M$ tori on a given energy level is even, and their significant feature is that they are locally stable for variations of all parameters, including B. Figure 2 illustrates the special case in a "thick" Fermi surface. If "fluted cylinders" are also present, then they also lie in the plane $\Gamma(\mathbf{B})$. These properties lead to the experimental predictions formulated above. Figure 3 illustrates the experimentally observed situation.

In closing, we wish to thank L. A. Fal'kovskil for a consultation on solid-state physics and Michael E. Fisher for assistance and helpful suggestions.

\section{REFERENCES}

[1] I. M. Lifshitz and V. G. Peschanskiı̌, Zh. Éksp. Teor. Fiz. 35, 1251 (1958) [Sov. Phys. JETP 8, 875 (1959)].

[2] E. M. Lifshitz and L. P. Pitaevskiı̌, Physical Kinetics, Pergamon Press, New York, 1981 [Russian original, Nauka, Moscow, 1979], Chapter 9, § 84.

[3] C. Kittel, Quantum Theory of Solids, John Wiley, New York, 1963.

[4] I. M. Lifshits, M. Ya. Azbel', and M. I. Kaganov, Electron Theory of Metals, Consultants Bureau, New York, 1973 [Russian original, Nauka, Moscow, 1971].

[5] S. P. Novikov, Usp. Mat. Nauk 37, 3 (1982).

[6] A. V. Zorich, Usp. Mat. Nauk 39, 235 (1984).

[7] S. P. Novikov, in Proceedings of the Conference on Topological Methods in Mathematics, dedicated to the 60th birthday of J. Milnor, June 15-22, 1991, SUNY Stony Brook, 1993.

[8] I. A. Dynnikov, Mat. Zametki 53, 57 (1993).

[9] S. P. Novikov, in Proceedings of the Conference on Geometry, December 15-26, 1993, Tel Aviv University, 1995 (in press).

[10] S. P. Tsarev and I. A. Dynnikov, Private communication (1992-93).

Institute for Physical Science and Technology, University of Maryland, College Park, Maryland 20742-2431

E-mail address: novikov@ipst.umd.edu, novikov@landau.ac.ru

L. D. Landau Institute of Theoretical Physics, Russian Academy of Sciences, 117940 Moscow, Russia 